\title{
Prevalência de disfunção temporomandibular e ansiedade em estudantes universitários
}

Prevalence of temporomandibular dysfunction and anxiety in university students

Prevalencia de disfunción temporomandibular y ansiedad en estudiantes universitarios

Bruna Rodrigues BARRETO

Curso de Odontologia, Faculdade Santa Maria, Departamento de Odontologia. 58900-000, Cajazeiras - PB, Brasil

Clarissa Lopes DRUMOND

Docente do Curso de Odontologia, Faculdade Santa Maria, 58900-000, Cajazeiras- PB, Brasil https://orcid.org/0000-0001-8944-852X

Rodolfo de Abreu CAROLINO

Docente do Curso de Odontologia, Faculdade Santa Maria, 58900-000, Cajazeiras- PB, Brasi https://orcid.org/0000-0002-7962-024X

José Klidenberg de OLIVEIRA JÚNIOR

Docente do Curso de Odontologia, Faculdade Santa Maria, 58900-000, Cajazeiras- PB, Brasil https://orcid.org/0000-0002-4539-2007

\section{Resumo}

Introdução: A Disfunção Temporomandibular (DTM) consiste em alterações fisiológicas da Articulação Temporomandibular (ATM), que está inserida no sistema estomatognático. O diagnóstico inicial pode ser difícil devido à complexidade da ATM e sua relação com as demais estruturas anatômicas da face. Objetivos: Avaliar a prevalência de sinais e sintomas de disfunção temporomandibular e ansiedade em alunos dos cursos da área de saúde (Biomedicina, Farmácia, Fisioterapia, Nutrição, Medicina e Odontologia) da Faculdade Santa Maria, Cajazeiras, Paraíba, Brasil. Métodos: Essa pesquisa caracteriza-se como transversal, de natureza exploratória e de caráter descritivo/quantitativo, foram avaliados 130 universitários. Com o intuito de investigar possíveis associações entre a DTM e a ansiedade foram empregados questionários estruturados e validados na literatura. Com relação a avaliação dos sinais e sintomas da DTM e da ansiedade foram selecionados dois instrumentos, respectivamente: O Índice de Fonseca que avalia sinais e sintomas de DTM e o Índice de Ansiedade Traço-Estado (IDATE). Resultados: Os dados obtidos indicam que a maioria da amostra é composta por estudantes do curso de odontologia (80\%). Além disso, 59\% dos alunos apresentou algum grau de disfunção, tendo a maioria como grau leve. O IDATE mais prevalente foi o grau moderado. Conclusão: Conclui-se que houve uma alta prevalência de disfunção temporomandibular e ansiedade nos estudantes de odontologia, enquanto nos demais cursos ficaram entre leve e moderada. Sugere-se que uma abordagem terapêutica multidisciplinar seja realizada para os grupos estudados, tendo em vista o caráter multifatorial das desordens temporomandibulares.

Descritores: Ansiedade; Articulação Temporomandibular; Dor Facial.

\section{Abstract}

Introduction: Temporomandibular Dysfunction (TMD) consists of physiological changes in the Temporomandibular Joint (TMJ), which is inserted in the stomatognathic system. The initial diagnosis can be difficult due to the complexity of the TMJ and its relationship with the other anatomical structures of the face. Objectives: To evaluate the prevalence of signs and symptoms of temporomandibular disorders and anxiety in students of health courses (Biomedicine, Pharmacy, Physiotherapy, Nutrition, Medicine and Dentistry) at Faculdade Santa Maria, Cajazeiras, Paraíba, Brazil. Methods: This research is characterized as cross-sectional, exploratory and descriptive / quantitative, 130 university students were evaluated. In order to investigate possible associations between TMD and anxiety, structured questionnaires were used and validated in the literature. Regarding the assessment of TMD signs and symptoms and anxiety, two instruments were selected, respectively: the Fonseca Index that assesses TMD signs and symptoms and the State-Trait Anxiety Index (STAI). Results: The data obtained indicate that the majority of the sample is made up of dentistry students (80\%). In addition, $59 \%$ of students had some degree of dysfunction, with the majority having a mild degree. The most prevalent STAI was the moderate grade. Conclusion: We conclude that there was a high prevalence of temporomandibular disorders and anxiety in dentistry students, while in other courses they were between mild and moderate. It is suggested that a multidisciplinary therapeutic approach be carried out for the groups studied, in view of the multifactorial character of temporomandibular disorders.

Descriptors: Anxiety; Temporomandibular Joint; Facial Pain.

\section{Resumen}

Introducción: La disfunción temporomandibular (TMD) consiste en cambios fisiológicos en la articulación temporomandibular (TMJ), que se inserta en el sistema estomatognático. El diagnóstico inicial puede resultar difícil debido a la complejidad de la ATM y su relación con el resto de estructuras anatómicas del rostro. Objetivos: Evaluar la prevalencia de signos y síntomas de trastorno temporomandibular y ansiedad en estudiantes de cursos de salud (Biomedicina, Farmacia, Fisioterapia, Nutrición, Medicina y Odontología) de la Faculdade Santa Maria, Cajazeiras, Paraíba, Brasil. Métodos: Esta investigación se caracteriza por ser transversal, exploratoria y descriptiva / cuantitativa, se evaluaron 130 estudiantes universitarios. Para investigar posibles asociaciones entre TMD y ansiedad, se utilizaron cuestionarios estructurados y se validaron en la literatura. En cuanto a la evaluación de los signos y síntomas de los TTM y la ansiedad, se seleccionaron dos instrumentos, respectivamente: el Índice de Fonseca que evalúa los signos y síntomas de los TTM y el Índice de Ansiedad Rasgo-Estado (STAI). Resultados: Los datos obtenidos indican que la mayoría de la muestra está formada por estudiantes de la carrera de Odontología (80\%). Además, el $59 \%$ de los estudiantes presentaba algún grado de disfunción y la mayoría presentaba un grado leve. El STAI más prevalente fue el grado moderado. Conclusión: Se concluye que hubo una alta prevalencia de trastornos temporomandibulares y ansiedad en los estudiantes de Odontología, mientras que en otros cursos estuvieron entre leves y moderados. Se sugiere realizar un abordaje terapéutico multidisciplinario para los grupos estudiados, dado el carácter multifactorial de los trastornos temporomandibulares.

Descriptores: Ansiedad; Articulación Temporomandibular; Dolor Facial.

INTRODUÇÃO

As articulações temporomandibulares (ATMs) são junturas do tipo sinovial que estão inseridas no sistema estomatognático, e responsáveis pela função mastigatória, da fala, deglutição e respiração ${ }^{1}$. Esta articulação pode ser acometida por fatores extrínsecos e intrínsecos, gerando alterações musculoesqueléticas, conhecidas como Desordens Temporomandibulares (DTM), essas alterações podem englobar os músculos mastigatórios, bem como as estruturas 
anatômicas da $\mathrm{ATM}^{2}$. De acordo $\mathrm{com}^{1}$ os distúrbios que envolvem os músculos mastigatórios são os relativos às DTM musculares, já os que envolvem os distúrbios e as alterações na ATM, são classificadas como articulares.

A etiologia da DTM é considerada complexa e multifatorial, sendo associada a fatores predisponentes, iniciadores e perpetuantes, tais como: alterações oclusais, hábitos parafuncionais, estresse, ansiedade, anormalidades no disco intra-articular, do complexo côndilo-disco, da membrana sinovial e cápsula articular ${ }^{3}$. Tais fatores podem estar relacionados à ocorrência de inflamações articulares, dores musculares ou espasmos ${ }^{4}$.

Vários são os trabalhos científicos que avaliam 0 crescente aumento das dores relacionadas às disfunções temporomandibulares, classificando atualmente como um problema de saúde pública, tendo em vista que, afetam os indivíduos de forma social, cultural e psicológica ${ }^{5}$.

Sugere-se que entre as causas dessas disfunções estão as psicossomáticas. Os fatores emocionais, como ansiedade, estresse e depressão podem desencadear hábitos parafuncionais (como onicofagia, mastigação unilateral e bruxismo) e tensão muscular, levando ao surgimento dos sinais e sintomas das DTM ${ }^{6}$.

A ansiedade é uma experiência emocional em função da previsão de situações futuras consideradas desagradáveis pelo indivíduo. Assim, avaliações, seminários e trabalhos em grupo podem vir a gerar pensamentos desagradáveis relacionados aos processos, fazendo emergir essa ansiedade ${ }^{7}$.

Os estudantes da área de saúde apresentam diferentes níveis de ansiedade, que proporcionalmente aumentam ao longo do curso, tal fato justifica-se pelo ritmo intenso de estudos agregado à falta de reconhecimento da sobrecarga de estresse emocional e físico. Como também a ausência de acompanhamento psicológico, o que resulta no aparecimento de diversos distúrbios, que além de ter efeito sob o emocional do indivíduo, demonstram sinais físicos no corpo, como as desordens temporomandibulares ${ }^{8}$.

É válido investigar e identificar os grupos que podem apresentar fatores predisponentes para 0 desenvolvimento das DTMs. Dessa forma, o diagnóstico realizado por meio de instrumentos validados pela comunidade científica torna-se imprescindível, possibilitando um panorama epidemiológico do problema.

O objetivo do presente estudo foi avaliar a prevalência de DTM e a sintomatologia da disfunção temporomandibular relacionada a ansiedade em estudantes dos cursos da área de saúde (Biomedicina, Enfermagem Farmácia, Fisioterapia, Nutrição, Psicologia, Medicina e Odontologia) da Faculdade Santa Maria, Cajazeiras, Paraíba, Brasil.

MATERIAL E MÉTODO

- Tipo de estudo

Esse estudo caracteriza como transversal, com 0 intuito de identificar eventuais diferenças estatísticas, adotando como estratégia um questionário especifico.

- Cenário da pesquisa

O município de Cajazeiras - Paraíba (PB) está localizando no sertão paraibano, distanciando-se da capital João Pessoa - PB por $475 \mathrm{~km}$, e possuindo 61.776 habitantes. Para este trabalho, selecionou-se a Faculdade Santa Maria- Instituição de Ensino Superior (IES) privada para realização da pesquisa.

O universo será composto por alunos regularmente matriculados do $1^{\circ}$ ao $10^{\circ}$ período dos cursos da área da saúde da referida IES, na cidade de Cajazeiras-PB, durante o período de agosto de 2020 a outubro de 2020.

- Tamanho amostral

Para realização do cálculo amostral utilizou-se os parâmetros estatísticos fundamentais (número absoluto da população que foi 1.750 , margem de erro amostral de 5\%, nível de confiança $95 \%$ e a forma de distribuição da população). Para tanto, utilizou-se uma magnitude de efeito alta $(0,8)$ de acordo com o ranqueamento de Cohen, escore Z tipo I $(1,64)$ unicaudal, gerando um poder de $80 \%$. Dessa forma, levando em consideração do tamanho da população $(n=1750)$, após ajustes gerou-se o tamanho amostral de $n=120$, considerando um $p$ estatisticamente significativo $(p=0,05)$. Para realização do cálculo amostral foi utilizado o programa Microsoft® Office Excel 2010

- Critérios de inclusão

Para a participação dos graduandos nessa pesquisa foram considerados como critérios de inclusão: ser discente da área da saúde do $1^{\circ}$ ao $10^{\circ}$ período da Faculdade Santa Maria, constar matrícula ativa na listagem concedida pela secretaria acadêmica da Instituição de Ensino Superior - IES solicitada por meio da Carta de Anuência, bem como autorizar sua participação pelo Termo de Consentimento Livre e Esclarecido (TCLE), o qual foi realizado de maneira remota, assim como toda a pesquisa.

- Critérios de exclusão

Foram excluídos da pesquisa os estudantes que apresentaram as seguintes 
características: questionário discentes submetidos a psicológicos/psiquiátricos,

incompleto, tratamentos alterações sistêmicas, participantes que tivesse fazendo uso de antidepressivos/ansiolítico, cirurgia ortognática, discentes não cooperantes.

- Instrumento e procedimento de coleta de dados

A coleta de dados foi realizada de forma remota entre os meses de setembro e outubro de 2020 por um pesquisador devidamente treinado.Três questionários estruturados anonimamente direcionados aos discentes foram enviados via Google Forms ${ }^{\circledR}$ disponibilizando um link por e-mail e/ou via wahtsaap da referida IES. Para a avaliação dos sinais e sintomas da DTM e de ansiedade foram selecionados dois instrumentos: O Índice de Fonseca e o Inventário de Ansiedade TraçoEstado (IDATE).

O Índice de Fonseca é um questionário validado e utilizado em pesquisas desta categoria, que demonstrou excelente confiabilidade e elevada acurácia para o diagnóstico da DTM de origem muscular. $O$ instrumento inclui informações a respeito da dificuldade em abrir a boca e movimentar a mandíbula; dores de cabeça, na nuca, pescoço ou regiões articulares, ruído nas articulações temporomandibulares, hábito de apertar ou ranger os dentes. É composto por 10 perguntas para as quais as possíveis respostas são sim (10 pontos), as vezes (5 pontos) e não (0 pontos). Para cada pergunta, somente pode ser assinalada uma resposta. A soma dos pontos é usada para classificar a severidade da $\mathrm{DTM}^{9}$ :

- Sem DTM (de 0 a 15 pontos)

- DTM leve (de 20 a 40 pontos)

- DTM moderada (de 45 a 65 pontos)

- DTM severa (de 70 a 100 pontos) Inventário de Ansiedade Traço-Estado IDATE: Esse instrumento é constituído de 40 afirmações a respeito dos sentimentos do sujeito, distribuídas em duas partes. A primeira parte avalia a ansiedade-estado, enquanto a segunda avalia a ansiedade-traço. Cada parte consiste de 20 afirmações descritivas de sentimentos pessoais, as quais os indivíduos pontuam com base na intensidade da ansiedade que está ocorrendo naquele momento (ansiedade-estado) ou com base na frequência com esses sentimentos ocorrem (ansiedadetraço), por meio de uma escala que varia de $1 \mathrm{a}$ 4 pontos.Cada questão possui escores de 1 a 4 , sendo que o total varia de 20 a 80 para cada escala e demostram: 20 a 34 (ansiedade baixa), 35 a 49 (ansiedade moderada), 50 a 64 (ansiedade elevada) e 65 a 80 (ansiedade muito elevada). Nas duas escalas do Inventário de Ansiedade Traço-Estado (IDATE), os escores altos indicam mais ansiedade ${ }^{10}$.

- Considerações éticas

De acordo com a resolução CNS 510/16, este projeto foi submetido à apreciação pelo Comitê de Ética em Pesquisa da Faculdade Santa Maria - FSM e teve aprovação sob $n^{\circ}$ do parecer: 4.361.928. Os discentes foram inicialmente informados sobre os objetivos da pesquisa e todas as suas dúvidas serão esclarecidas. Estando os mesmos de acordo com a sua participação, assinaram o Termo de Consentimento Livre e Esclarecido, o qual garantia aos estudantes participantes da pesquisa a preservação do seu anonimato, bem como a inexistência de ônus decorrente da participação no estudo.

- Análise estatística

Após coletados, os dados foram inseridos no banco de dados do programa de informática Estatistical Package for Social Sciences (SPSS) para Windows, na versão 22.0, e analisados pela técnica de estatística descritiva e inferencial, adotando-se um intervalo de confiança de 95\%. As análises descritivas de frequências absoluta e relativa dos dados foram empregados para verificar a distribuição da amostra.

RESULTADOS

$$
\text { Participaram da pesquisa } 130
$$

universitários, sendo que a grande maioria dos discentes entrevistados foram do curso de odontologia $(80 \%)$ seguido do curso de biomedicina $(9,1 \%)$, medicina $(6,5 \%)$ e psicologia $(6,5 \%)$. Os períodos analisados apresentaram variação entre $01^{\circ}$ e $10^{\circ}$ do semestre da graduação, obtendo, portanto, a maior frequência de alunos matriculados no $10^{\circ}$ semestre.

Os discentes do curso de biomedicina e psicologia apresentaram, de acordo com os resultados do questionário IDATE, ansiedade moderada. Vale ressaltar que, os alunos do curso de Odontologia apresentaram uma variação entre as variáveis analisadas, os quais apresentou índices de ansiedade elevada (12\%) e (4\%) para ansiedade leve. O curso de medicina apresentou tanto grau de ansiedade moderada $(80 \%)$ como também para ansiedade elevada $20 \%$ (Tabela 1).

De acordo com e o formulário IDATE (ESTADO) podemos observar que $100 \%$ dos estudantes participantes dos cursos de fisioterapia e nutrição apresentaram ansiedade elevada, enquanto que, $60 \%$ da amostra analisada dos cursos de medicina, odontologia 
e psicologia apresentaram ansiedade moderada (Tabela 2).

Tabela 1. Resultados obtidos no instrumento de avaliação para ansiedade. IDATE - TRAÇO

\begin{tabular}{l|l|l|l|l|l|l}
\hline \multirow{2}{*}{ Qual o seu curso? } & \multicolumn{7}{|c}{ IDATE - TRAÇO } \\
\cline { 2 - 8 } & \multicolumn{2}{|c|}{ Ansiedade Baixa } & \multicolumn{2}{|c}{ Ansiedade Moderada } & \multicolumn{2}{c}{ Ansiedade Elevada } \\
\hline Biomedicina & 0 & $0,0 \%$ & 7 & $100,0 \%$ & 0 & $0,0 \%$ \\
\hline Farmácia & 1 & $25,0 \%$ & 3 & $75,0 \%$ & 0 & $0,0 \%$ \\
\hline Medicina & 0 & $0,0 \%$ & 4 & $80,0 \%$ & 1 & $20,0 \%$ \\
\hline Nutrição & 1 & $50,0 \%$ & 1 & $50,0 \%$ & 0 & $0,0 \%$ \\
\hline Odontologia & 5 & $4,8 \%$ & 86 & $82,7 \%$ & 13 & $12,5 \%$ \\
\hline Psicologia & 0 & $0,0 \%$ & 5 & $100,0 \%$ & 0 & $0,0 \%$ \\
\hline Fisioterapia & 1 & $33,3 \%$ & 2 & $66,7 \%$ & 0 & $0,0 \%$ \\
\hline
\end{tabular}

Tabela 2. Resultados obtidos no instrumento de avaliação para ansiedade. IDATE - ESTADO

\begin{tabular}{|c|c|c|c|c|c|c|c|c|}
\hline \multirow{3}{*}{$\begin{array}{l}\text { Qual o seu } \\
\text { curso? }\end{array}$} & \multicolumn{8}{|c|}{ IDATE - ESTADO } \\
\hline & \multicolumn{2}{|c|}{$\begin{array}{c}\text { Ansiedade } \\
\text { Baixa }\end{array}$} & \multicolumn{2}{|c|}{$\begin{array}{l}\text { Ansiedade } \\
\text { Moderada }\end{array}$} & \multicolumn{2}{|c|}{$\begin{array}{c}\text { Ansiedade } \\
\text { Elevada }\end{array}$} & \multicolumn{2}{|c|}{$\begin{array}{c}\text { Ansiedade } \\
\text { muito Elevada }\end{array}$} \\
\hline & 0 & $0,0 \%$ & 6 & $85,7 \%$ & 1 & $14,3 \%$ & 0 & $0,0 \%$ \\
\hline Farmácia & 0 & $0,0 \%$ & 4 & $100,0 \%$ & 0 & $0,0 \%$ & $\mathrm{o}$ & $0,0 \%$ \\
\hline Medicina & 0 & $0,0 \%$ & 3 & $60,0 \%$ & 2 & $40,0 \%$ & 0 & $0,0 \%$ \\
\hline Nutrição & 0 & $0,0 \%$ & 0 & $0,0 \%$ & 2 & $100,0 \%$ & 0 & $0,0 \%$ \\
\hline Odontologia & 2 & $1,9 \%$ & 63 & $60,6 \%$ & 36 & $34,6 \%$ & 3 & $2,9 \%$ \\
\hline Psicologia & 0 & $0,0 \%$ & 3 & $60,0 \%$ & 2 & $40,0 \%$ & 0 & $0,0 \%$ \\
\hline Fisioterapia & 0 & $0,0 \%$ & 0 & $0,0 \%$ & 3 & $100,0 \%$ & 0 & $0,0 \%$ \\
\hline
\end{tabular}

De acordo com a associação dos Índices de Fonseca e o Formulário IDATE (TRAÇO) pode-se observar que $90 \%$ dos estudantes participantes apresentaram DTM moderada e severa associado a uma ansiedade moderada, e aqueles que não foram diagnosticados com DTM apresentam uma maior prevalência para o grau de ansiedade moderada (73,6\%) (Tabela 3).

Tabela 3. Associação entre IDATE - Traço e Índice Fonseca

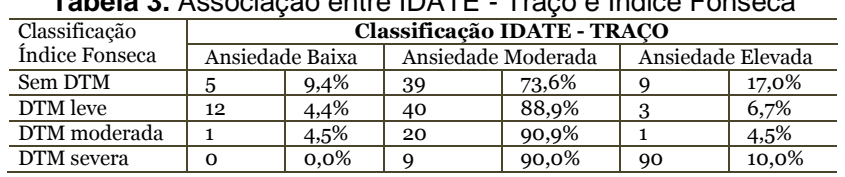
De acordo com a associação dos Índices de Fonseca e IDATE - Estado, observa-se que $64 \%$ dos participantes apresentaram ansiedade moderada sem indícios para DTM de acordo com os instrumentos utilizados. Foi possível evidenciar que aqueles que apresentaram DTM moderada $(63 \%)$ demonstram ter ansiedade moderada, enquanto que, $36 \%$ possuem ansiedade elevada e DTM moderada. Ainda foi possível constatar que grande parte dos alunos entrevistados possuem algum grau de DTM e essa condição sempre está associada a algum grau de ansiedade.

De acordo com os achados da pesquisa, com relação a traços de ansiedade, somente os cursos de medicina (20\%) e de odontologia $(12,5 \%)$ apresentaram níveis de ansiedades elevados, os demais cursos se enquadram nas classificações entre baixa e moderada (Tabela 4).

Tabela 4. Associação entre IDATE - Estado e Índice Fonseca

\begin{tabular}{l|c|c|c|c|c|c|c|c}
\hline \multirow{2}{*}{ Índice Fonseca } & \multicolumn{6}{c|}{ IDATE - ESTADO } \\
\cline { 2 - 9 } & $\begin{array}{c}\text { Ansiedade } \\
\text { Baixa }\end{array}$ & \multicolumn{2}{c}{$\begin{array}{c}\text { Ansiedade } \\
\text { Moderada }\end{array}$} & \multicolumn{2}{c}{$\begin{array}{c}\text { Ansiedade } \\
\text { Elevada }\end{array}$} & \multicolumn{2}{c}{$\begin{array}{c}\text { Ansiedade } \\
\text { muito Elevada }\end{array}$} \\
\hline Sem DTM & 1 & $1,9 \%$ & 34 & $64,2 \%$ & 18 & $34,0 \%$ & 0 & $0,0 \%$ \\
\hline DTM leve & 1 & $2,2 \%$ & 27 & $60,0 \%$ & 15 & $33,3 \%$ & 2 & $4,4 \%$ \\
\hline DTM moderada & 0 & $0,0 \%$ & 14 & $63,6 \%$ & 8 & $36,4 \%$ & 0 & $0,0 \%$ \\
\hline DTM severa & 0 & $0,0 \%$ & 4 & $40,0 \%$ & 5 & $50,0 \%$ & 1 & $10,0 \%$ \\
\hline
\end{tabular}

DISCUSSÃO

O diagnóstico precoce das DTM é fundamental para resolutividade e melhora do quadro de sintomatologia do paciente. Dessa forma, a utilização de instrumentos validados e disponíveis na literatura tem sido largamente utilizado, pois são de fácil aplicação e interpretação auxiliando na consolidação do diagnóstico ${ }^{11}$.

Faz-se necessário ressaltar a influência das doenças psicossomáticas sobre 0 comportamento da dor na musculatura mastigatória e relacioná-la aos achados subjetivos da dor. No que tange aos grupos mais expostos, os acadêmicos universitários tem sido alvo de estudo, justamente pela intensa pressão relacionado a cobrança durante a fase universitária ${ }^{12}$.

No presente estudo optou-se por avaliar a prevalência de sinais e sintomas de DTM e ansiedade em uma amostra de universitários de todos os períodos disponíveis nos respectivos cursos da instituição escolhida. Tal escolha, justifica-se pela escassez de estudos mais aprofundados relacionados a DTM e ansiedade nesta amostra específica que está exposta a uma elevada carga emocional, incluindo a ansiedade, estresse e excesso de cobrança, dando espaço para o aparecimento de outras doenças $^{13 .}$ Os estudos recentes apontam que os fatores emocionais estão intimamente relacionados ao aparecimento e progressão da DTM $^{8}$

A prevalência de DTM entre os discentes da área de saúde da FSM correspondeu a 59\%, assemelhando-se ao estudo de Patrocínio et al. ${ }^{8}$ o qual obteve uma prevalência de $78 \%$ e Medeiros t al. ${ }^{14}$ e Fernandes et al. ${ }^{15}$ com uma prevalência de $74,9 \%$ e $75 \%$, respectivamente. Em relação os possíveis graus de DTM aferidos no instrumento, o que predominou foi o leve, seguido do moderado e severo, esse dado corrobora com a ordem de severidade dos estudos citados.

A literatura tem mostrado uma associação positiva entre diferentes fatores emocionais e a presença de sinais e sintomas de DTM. ${ }^{15-19}$ Nesse estudo, o relato de ansiedade foi estatisticamente associado à presença da sintomatologia da DTM, estando estes achados de acordo com outros estudos realizados em diferentes grupos. Dessa forma, acredita-se que o grau de ansiedade pode afetar os processos biológicos de transmissão e percepção da dor, e ao longo prazo promover danos as ATMs e estruturas anatômicas associadas.

Vale ressaltar que a DTM vem se tornando cada vez mais frequente nos cursos de ensino superior ${ }^{8,20}$ exigindo uma atenção 
maior pela realização do diagnóstico precoce e até mesmo por parte dos familiares e das próprias faculdades em indicar ajuda psicológica para os acadêmicos que apresentem quadro de ansiedade, tendo em vista que essa condição é um fator que predispõe o aparecimento das DTM.

O quadro da classificação de traço e ansiedade mostraram que 0 curso de odontologia apresentou o nível de ansiedade classificada "muito elevada". Já os demais cursos apresentaram variações entre os três níveis (baixo, moderado e elevado). Já o curso de farmácia, por exemplo, teve uma prevalência de ansiedade moderada, enquanto que nutrição e fisioterapia tiveram ansiedade elevada. Uma das possíveis hipóteses que pode explicar essa frequência de ansiedade entre os cursos estudos, é justamente o excesso de pressão e inseguranças com relação a futura vida profissional afetando de forma negativa e ocasionando prejuízos para a vida desses universitários ${ }^{20,21}$.

A elevada prevalência de ansiedade autorrelatada, observada no presente estudo reforça a evidência da carga emocional excessiva ao qual os universitários estão submetidos, e essa condição está estatisticamente associados à presença da sintomatologia da DTM, o que pode sugerir que esse grupo é submetido a importantes fatores de risco para o aparecimento e progressão dessas desordens ${ }^{22}$.

É importante ressaltar as limitações do presente estudo, o momento atual da pandemia da COVID-19 dificultou a aplicação dos questionários, que precisou ser desenvolvido de forma remota e isso dificultou a adesão dos participantes. A obtenção dos dados torna-se limitado devido aos diagnósticos serem realizadas somente com formulários, sugerindo que os próximos estudos sejam desenvolvidos sobre uma perspectiva de avaliação epidemiológica e clínica.

CONCLUSÃO

Conclui-se que a amostra estudada apresenta uma alta prevalência de DTM. O nível de ansiedade mais prevalente foi o moderado, tanto na ansiedade traço como estado e a mesmas apresentam associação com a DTM nos acadêmicos da área da saúde da FSM. Sugerese que uma abordagem terapêutica multidisciplinar seja realizada para os grupos estudados tendo em vista o caráter multifatorial das desordens temporomandibulares.

REFERÊNCIAS

1. Chiodelli L, Pacheco AB, Missau TS, Silva AMT, Corrêa ECR. Associação entre funções estomatognáticas, oclusão dentária e sinais de disfunção temporomandibular em mulheres assintomáticas. Rev CEFAC.2015;17(1):117-25.

2. Greene CS, Klasser GD, Epstein JB. Revision of the American Association of Dental Rescarch's science information statement about temporomandibular disorders. J Can Dent Assoc. 2010:76-115.

3. Ohrbach R, Dworkin SF. The evolution of TMD diagnosis: past, present, future. J Dent Res. 2016;95(10):1093-101.

4. Melo Júnior $P C$, Aroucha JMCNL, Arnaud M, Lima MGDS, Gomes SGF, Ximenes R et al. Prevalence of TMD and level of chronic pain in a group of Brazilian adolescents. PloS One.2019;14(2):0205874.

5. Wieckiewicz $M$, Boening $K$, Wiland $P$, Shiau YY, Paradowska-Stolarz A. Reported concepts for the treatment modalities and pain management of temporomandibular disorders. J Headache Pain. 2015;16(1):1-12.

6. Velly AM, Look JO, Carlson C, Lenton PA, Kang W, Holcroft CA, Fricton JR. The effect of catastrophizing and depression on chronic pain-a prospective cohort study of temporomandibular muscle and joint pain disorders. Pain. 2011; 152(10):2377-383.

7. Coelho AT, Lorenzini LM, Suda EY, Rossini S, Reimão R. Qualidade de sono, depressão e ansiedade em universitários dos últimos semestres de cursos da área da saúde. Neurobiol.2010;73(1):35-9.

8. Patrocínio RT, Moura AC, Silva E, Carneiro MS, Tenôrio GM, Machado da Costa $\mathrm{CH}$. Disfunção temporomandibular e ansiedade em graduandos de Odontologia. Rev Cubana Estomatol. 2019;56(1):42-51.

9. Fonseca DM, Bonfante G, Valle A, Freitas SFT. Diagnóstico pela Anamanese da Disfunção Craniomandibular. RGO. 1994;42:23-8.

10. Spielberger CD, Gorsuch RL, Lushene RE. Manual for the state-trait anxiety inventory. New York: Consulting Psychologists; 1970.

11. Ichesco E, Quintero A, Clauw DJ, Peltier S, Sundgren PM, Gerstner GE et al. Altered functional connectivity between the insula and the cingulated cortex in patients with temporomandibular disorder: a pilot study. Headache. 2012;52(3):441-54.

12. Jesus LA, Martins MD, Andrade DV, BiasottoGonzalez DA. Estudo transversal da prevalência de disfunção temporomandibular e bruxismo em estudantes universitários. Rev Ter Man. 2009;7(29):11-4.

13. Guhur MLP, Alberto RN, Carniatto $N$. Influências biológicas, psicológicas e sociais do vestibular na adolescência. Roteiro 2010; 35(1):115-38

14. Medeiros SP, Batista AUD, Forte FDS. Prevalência de sintomas de Disfunção Temporomandibular e hábitos parafuncionais 
em estudantes universitários. Rev Gauch Odontol. 2011;59(2):201-8.

15. Fernandes AUR, Garcia AR, Zuim PRJ, Cunha LDP, Marchiori AV. Desordem temporomandibular e ansiedade em graduandos de odontologia. Ciênc Odontol Bras. 2007;10(1):70-7.

16. Monteiro DR, Zuim PRJ, Pesqueira AA, Ribeiro PP, Garcia AR. Relationship between anxiety and chronic orofacial pain of Temporomandibular Disorder in a group of university students. J Prosthodont Res. 2011; 55(3):154-58.

17. Giannakopoulos NN, Keller L, Rammelsberg P, Kronmüller KT, Schmitter M.Anxiety and depression in patients with chronic temporomandibular pain and in controls. J Dent. 2010;38(5):369-76.

18. Mottaghi A, Razavi SM, Elham Zamani Pozveh $\mathrm{E}$, Jahangirmoghaddam M. Assessment of the relationship between stress and temporomandibular joint disorder in female students before university entrance exam (Konkour exam). Dent Res J (Isfahan). 2011; 8(Supl. 1):76-9.

19. Pizolato RA, Freitas-Fernandes FS, Gavião MB. Anxiety/depression and orofacial myofacial disorders as factors associated with TMD in children. Braz Oral Res. 2013; 27(2):156-62.

20. Godinho DCA, Melo SR, Lemos MES, Furlan RMMM. Correlação entre sintomas de disfunção temporomandibular, hábitos orais deletérios e sintomas de estresse em estudantes universitários. Distúrbios da Comunicação, 2019;31(3):481-92.

21. Paulino MR, Moreira VG, Lemos GA, Silva PLPD, Bonan PRF, Batista AUD. Prevalência de sinais e sintomas de disfunção temporomandibular em estudantes prévestibulandos: associação de fatores emocionais, hábitos parafuncionais e impacto na qualidade de vida. Ciênc Saúde Coletiva. 2018;23(1):173-86.

22. Bezerra BPN, Ribeiro AIAM, Farias ABL, Farias $A B L$, Fontes LBC, Nascimento SR et al. Prevalência da disfunção temporomandibular e de diferentes níveis de ansiedade em estudantes universitários. Rev Dor. 2012;13(3):235-42.

\section{CONFLITO DE INTERESSES}

Os autores declaram não haver conflitos de interesse

AUTOR PARA CORRESPONDÊNCIA

José Klidenberg de Oliveira Júnior

Departamento de Odontologia,

Faculdade Santa Maria - FSM

58900-000 Cajazeiras- PB, Brasil

E-mail: joseklidemberg@gmail.com 